\title{
Empowerment Evaluation Applied: Experiences, Analysis, and Recommendations from a Case Study
}

\author{
CONNIE J. SCHNOES, VIRGINIA MURPHY-BERMAN, \\ AND JEFF M. CHAMBERS
}

\begin{abstract}
The empowerment evaluation approach is applied and examined within the context of a case study involving three comprehensive communities' initiatives. Although considerable literature has been published concerning the efficacy and legitimacy of the empowerment evaluation approach, little documentation exists regarding the empirical application of this approach. In this article we discuss, from both theoretical and empirical perspectives, issues related to the evaluator's role, differences relative to other inclusive evaluation approaches, stakeholder involvement, evaluation context variables, and the concept and practice of empowering program participants via an evaluation approach. Data were gathered to assess how the approach was viewed through the eyes of both the evaluation consumers and the evaluators. Recommendations are discussed regarding the types of circumstances that might most facilitate and support the empowerment evaluation approach.
\end{abstract}

\section{INTRODUCTION}

\section{Purpose of the Case Study}

The publication of Empowerment Evaluation (Fetterman, Kaftarian, \& Wandersman, 1996) sparked a re-examination of many longstanding discussions in the evaluation field. The purpose of the present paper is to explore, in the context of a case study, some of the issues raised in these discussions.

Evaluators are increasingly faced with an ever-growing body of literature focused on the strengths and weaknesses of diverse evaluation approaches. One key issue is how to select an evaluation approach that best fits the particular context within which one is working and that allows one to most effectively answer relevant evaluation questions. One particular context of interest is Comprehensive Community Initiatives (CCIs). CCIs represent new types of service intervention models for meeting the needs of children and families in communities. The CCI model emphasizes comprehensive, integrated, and collaborative ways of solving problems using community-based systems of service planning and delivery (Kubisch et al., 1995). As Kubisch (1996-7, p. 1) states:

Connie J. Schnoes • University of Nebraska at Lincoln, 2810 River Road Drive, Waterloo, NE 68069; Tel.: (402) 779-2814; E-mail: CJSchnoes@aol.com.

American Journal of Evaluation, Vol. 21, No. 1, 2000, pp. 53-64. All rights of reproduction in any form reserved. ISSN: $1098-2140$

Copyright (C) 2000 by American Evaluation Association. 
CCIs aim to do more than remediate problems .... . The change they seek is comprehensive, that is, inclusive of all sectors of the neighborhood-social, educational, economic, physical, and cultural environment. Change is focused on community building, that is, strengthening the capacity of neighborhood residents, associations and institutions.

We were selected to assist three CCIs in conducting program evaluations via a thirdparty contract with the state of Nebraska. As we considered our options for assisting projects in meeting their evaluation requirements, we examined several evaluation approaches, including the empowerment evaluation approach. Empowerment evaluation, as described by Fetterman, became our model of choice. We anticipated that by implementing the empowerment evaluation strategies, we would be able not only to provide projects with an effective first-year evaluation plan, but also to facilitate each project's ability to take increasing responsibility for the evaluation efforts as the programs evolved over time. The empowerment evaluation approach also seemed most responsive to the logistics of working with three projects that were diverse in their scopes of service, conceptual approaches to strengthening children and families, and geographic expanse and distance.

As we attempted to implement the empowerment model, we began to examine our efforts from several different perspectives. First, we began to discuss which aspects of the empowerment evaluation approach seemed to "best fit" our purposes and which aspects seemed to be less effective for achieving individual project objectives. We also found ourselves thinking about which features and strategies of empowerment evaluation appeared to be shared by other approaches (e.g., participatory, stakeholder-based, utilization-focused evaluation), and what seemed unique in the empowerment model. These discussions led to other inquiries. For instance, we considered how approaches to contextual issues, the evaluator's role, and decision-making appeared to influence the implementation of our empowerment approach. We reflected on how the evaluation framework that we developed to guide the direction of the evaluation and the development of evaluation questions appeared to affect implementation of our evaluation strategies. Finally, we questioned what makes an empowerment approach "empowering" in social program contexts such as the CCI framework in which we were working. Here, we present our reflections and observations about these issues, based on our work with the three CCI projects.

\section{Empowerment Evaluation: One Approach Among Many}

There has not been consistent agreement about the nature of the differences between empowerment evaluation and many other inclusive evaluation approaches such as stakeholder-based, participatory, and utilization-focused evaluation. Clearly, in application and design, these approaches share many common features, objectives, and strategies.

One key issue concerns the preferred role of the evaluator in the evaluation process. The role of the evaluator becomes increasingly critical when one considers its impact on the stakeholders (e.g., governing bodies, program managers, service providers, service recipients) and the decision-making processes. In his conceptualization of empowerment evaluation, Fetterman $(1996,1997)$ promotes close involvement between the evaluator and the program he or she is evaluating, because doing so is thought to produce more meaningful and useful evaluation results. Thus, in an empowerment evaluation model, the relationship between the evaluator and the evaluation consumers is characterized as a collaborative partnership. In this partnership, the evaluator seeks to involve project stakeholders in the 
evaluation process in meaningful ways. Collaboration refers to a relationship between the evaluator and the evaluation consumers in which the latter group is not only involved in the process, but control it. Thus, evaluation responsibilities are shared and the evaluation process is democratically managed (Fetterman, 1996). Meaningful participation includes, but is not limited to, planning and designing the evaluation, formulating evaluation questions, decisionmaking throughout the evaluation process, data analysis and collection, and the interpretation of evaluation results.

Empowerment evaluation's emphasis on promoting stakeholder involvement also is shared by other evaluation approaches such as stakeholder-based, participatory, and utilization-focused evaluation. For instance, Greene talks about collaboration in generating evaluation questions in her discussion of the evaluator's role in participatory evaluation (see Ryan, Greene, Lincoln, Mathison, \& Mertens, 1998). Folkman and Rai (1997) and Cousins and Earl (1992) discuss collaboration in participatory evaluation with respect to generating knowledge. Collaboration is also central to stakeholder-based evaluation, as stakeholders and evaluators work together to develop and finalize instruments and procedures, produce recommendations, and make decisions throughout the evaluation process (Ayers, 1987). Patton (1997a) further emphasizes collaborative decision-making structures in utilizationfocused evaluation.

In addition to stakeholder involvement, empowerment evaluation, participatory evaluation, and stakeholder-based evaluation share with utilization-focused evaluation a commitment to the meaningful use of results. All of these approaches emphasize the design of evaluation plans that result in the collection of data that is useful to the enhancement and refinement of program efforts and is meaningful in terms of examining program outcomes, benefits, and sustainability.

This similarity in emphasis on stakeholder involvement in decision-making and on results utilization in these approaches has led some, such as Patton, to question how empowerment evaluation differs at all from other collaborative forms of evaluation. Patton (1997a), in fact, suggests that many of the key concepts of empowerment evaluation, such as the importance of participation, collaboration, data usefulness, and coaching, are not unique to the empowerment evaluation approach. Patton further suggests that it is unclear how utilizing these collaborative techniques to "empower" individuals would differ from employing them simply as useful components of any good evaluation process. Sechrest (1997) asserts that empowerment is never really adequately defined in Fetterman's model and that the term empowerment lends itself to diverse interpretations. Who, Sechrest asks, is empowered to do exactly what?

Some have suggested that empowerment evaluation does differ from other collaborative evaluation approaches in the emphasis placed on stakeholder takeover of evaluation functions over time. Even here, the difference may be primarily one of degree. Patton (1997a), for instance, asserts that as a result of the collaborative process, all participatory evaluation approaches attempt to strengthen stakeholders' ability to sustain evaluation functions over time. However, empowerment evaluation makes stakeholder self-determination a more specific focus of the evaluation effort. In this regard, Fetterman $(1996,1997)$ discusses empowerment evaluation as a means to an end in enabling programs to become independent in their program evaluation efforts. He describes the empowerment evaluation process as consisting of four steps: 
1. Taking stock of concerns and resources, a process that includes all members of an organization or project.

2. Planning to establish a vision that all members are dedicated to, and developing strategies and action plans to achieve the vision.

3. Implementing strategies and action plans to facilitate the process.

4. Generating outcomes to document competence and progress relevant to goals or vision.

As a result of this empowerment evaluation process, Fetterman asserts that stakeholders should gain knowledge, training, experience, and insights critical to the technical aspects of conducting program evaluations, while simultaneously developing an appreciation for the usefulness and meaningfulness of the data generated. Thus, the empowerment evaluator's role as consultant, trainer, and facilitator should allow him to gradually disengage from the project's evaluation component as the stakeholders become more competent in and committed to the ongoing program evaluation. It was this aspect of the empowerment model, along with its collaborative focus, that seemed particularly suited to the community context in which we were working. Emphasis was on a "devolution" model of power and responsibility in which communities were encouraged to take increasing responsibility over time not just for program planning and implementation, but also for program evaluation and accountability.

\section{THE CASE STUDY}

\section{Evaluation Design}

In 1996 and 1997, the University of Nebraska's Center on Children, Families and the Law (CCFL) had the opportunity to contract with the state of Nebraska to provide evaluation services for three Comprehensive Community Initiatives (CCIs) funded by Family Preservation and Support grants. CCFL formulated a proposal to provide evaluation services to the three CCIs in response to a request for proposals (RFP) by the state, and was subsequently awarded the contract.

The three CCIs differed in their conceptual orientations as well as geographic locations. One CCI was composed of three counties and their respective coalitions in the western Nebraska panhandle. This CCI organized around a shared leadership approach with a focus on educating children and families and gathering for celebration and support. The second CCI was comprised of a two-census tract area of an urban community in eastern Nebraska. This CCI approached the empowerment of children and families by strengthening resources at the local level and by building on existing neighborhood strengths. The third CCI encompassed a single county and its seven communities in north-central Nebraska. The orientation for this CCI focused on enhancing parenting skills primarily through parent training instruction and resource materials.

CCFL introduced itself to the CCIs and initiated a working relationship. In early meetings, the evaluators, along with the state Technical Support Team, met with a diverse range of stakeholders at each project site to discuss possible evaluation strategies. After these opening meetings, our main contacts were with the project managers at each CCI site. Throughout the year we did continue also to periodically discuss our evaluation strategies and 
requested feedback about them from a wide group of people involved in the CCIs' projects, including individuals from community boards, planning teams, and neighborhood working groups.

The work of Roberts and Wasik (1996) most directly influenced the development of the general framework that guided the evaluation effort (see Murphy-Berman, Schnoes, \& Chambers, in press, for a fuller discussion of our evaluation model). This framework included three components: a contextual component, an approach component, and an outcomes component. The contextual component entailed investigating demographic and social organizational variables that shaped and defined the environmental context in which each CCI operated. The approach component included an examination of the CCI's underlying conceptual orientation to serving children and families, evaluation of organizational structures put in place to guide program development, and analysis of the specific programs and services offered. Outcomes were to be investigated at both the program-specific and project-wide level. Within this broad framework each project was to tailor the evaluation design and data collection strategies to best fit its particular interests and needs. It was within this tailoring process that we attempted utilization of the empowerment approach.

\section{The Complexities of Implementation}

The context of our involvement with the Nebraska Family Preservation Projects set the stage for the sequence of events that took place throughout our evaluation endeavors with the CCIs. One of the more prominent themes within the grant guidelines was for each project to demonstrate a "new way of doing business". Each project was to be creative in conceptualizing how to put in place new types of interventions to help families. Projects also were supposed to be community-based. Both of these requirements supported the use of an empowerment evaluation approach. It was believed that by working closely with each CCI, teaching them about evaluation, and demonstrating its usefulness to their particular needs, projects would be able to take over more and more of the responsibility for the evaluation process over time. Thus, we would empower the CCIs by facilitating their competence in and commitment to the evaluation components of the project.

Our first step was to initiate contact with each of the CCIs and begin to develop an identity as allies and team players. We approached each CCI from an empowerment evaluation orientation. Specifically, we stated that we were interested in working closely with each CCI to better understand the projects and thus guide them in conducting evaluations that addressed issues of relevance to them. We stressed the necessity for and value of open, honest communication while acknowledging the divergence of our approach from other more traditional, directive evaluation approaches. In addition to beginning to build the foundation of our working relationship, we were laying the groundwork for taking the first step in empowerment evaluation: taking stock (Fetterman, 1996). Our stock-taking activities included talking with the CCIs about their projects' conceptual orientations about empowering children and families within their communities; reading each CCI proposal carefully to become familiar with the CCI's assumptions and objectives; and sorting through archival material (e.g., activity logs, meeting minutes, previous needs assessment survey results) to gain an understanding of the "birth" of each CCI. Through these stock-taking activities we were not only attempting to identify resources and concerns, but also to understand and become familiar with the projects and the contexts in which they were being implemented. We continued stock-taking activities over the course of the next several months. In this effort, 
we looked beyond the principal stakeholders of the CCIs and gathered data about the context and social organization of each CCI community.

Somewhat simultaneously, we initiated step 2: planning. We asked questions in an effort to encourage the CCIs to think about where they were, where they wanted to end up, and what their expectations were for the projects. We were attempting to assist the CCIs in articulating their vision for their unique approach. Another component of step 2 is to develop strategies and action plans. To facilitate these activities, we offered consultation services to aid in the design of evaluation plans, instruments, and data collection tools, and in identifying potential sources of archival data. We asked questions such as, 1) What do you want to know about your project in six months? In a year?; 2) What particular outcomes are you most interested in achieving? Why?; 3) What do you think would be a reasonable timeframe and effective method of measuring the project's impact on these outcomes? To assist communities in thinking about how to approach their particular evaluation questions, step-by-step worksheets were constructed and made available to the CCIs. These gave participants the opportunity to identify and break apart their evaluation theories, objectives, and outcome goals. Participants were also provided with our overall evaluation framework and examples of potential data collection strategies that could be used to answer different types of evaluation questions within this framework.

To facilitate implementation, the third step of the empowerment evaluation process, we offered to provide training in evaluation techniques and methods, and to collect and analyze data. We also offered to collect and organize information from the CCIs that was being collected on an ongoing basis, such as meeting agendas and telephone logs. As we developed the survey that we used to assess the social context in which each CCI was operating, we sent draft forms of these surveys to the CCIs for their review. We asked for their input as we attempted to draft tools that we hoped would be helpful to their evaluation planning and implementation activities. We also established regular telephone conference calls at the frequency requested by the CCIs and we conducted site visits to attend board meetings, planning sessions, and training workshops. To help the CCIs think about evaluation issues from a broader perspective, other types of resources were offered. For instance, the CCIs were given access to an evaluation resource library that contained articles and papers about the experiences of other communities around the nation in evaluating a variety of types of comprehensive initiatives. An evaluation newsletter was started: participants from each of the three CCIs were invited to share their own ideas and thoughts about setting up and implementing various types of evaluation strategies. In this newsletter, an "article of the month" featured a summary of what we felt was a particularly outstanding article about some key CCI evaluation issue.

All of the above was done to facilitate adherence to the state's mandate for a rigorous results-based evaluation, while at the same time providing the CCIs with the means to meet this standard in a flexible manner that made sense to them in terms of their own project concerns. Our goal was to provide the CCIs with a range of resources that they could draw upon as they attempted to tailor an evaluation approach to their unique project circumstances. Thus, our concept of training was as a multidimensional process, and we saw ourselves as coaches or facilitators rather than as teachers or experts. We further felt that by offering a wide range of resources we could, as Fetterman (1996) suggests, best accommodate the evaluation to each project's particular local circumstances and to the degree of expertise possessed by each of our different evaluation clients. 


\section{Learning from Experience}

As suggested earlier, the empowerment approach presumes very active participation by evaluation clients in the evaluation process. It further assumes that clients want and see the need for evaluation of some type (or will develop this perspective), but feel that they need assistance in carrying out some aspects of the evaluation procedure effectively. Finally, in the empowerment approach, the assumption is that clients expect the results of the evaluation process to have real consequences in terms of decisions made about program development and program sustainability. These key assumptions were not fully supported in our particular case.

Initially, each of the CCIs responded in a positive, somewhat relieved, and hopeful manner to our approach. They expressed appreciation for our interest in actively including them in the evaluation process and becoming familiar with their projects and situations. They reported feeling optimistic about effecting change via their projects and about their working relationships with the state. In our early interactions, they had begun to share their ideas about desired outcomes and their notions of how they thought they would best achieve these outcomes.

As we followed through on agreed-upon meeting schedules, we anticipated that over time the CCIs would become more involved in the evaluation process and would increasingly bring up issues for us to consider at our meetings. In fact, we actively encouraged this and emphasized our desire to hear about their concerns and not to simply impose our agenda on them. This initiation of evaluation-related topics from the CCIs occurred with less frequency than we had hoped. In some instances, discussions simply became an opportunity for the program managers to conduct business with one another, which led to discussions relevant to the CCI management but not directly related to the immediate needs of the evaluation component. This occurred because some program managers were geographically separated from one another and had little opportunity to interact outside of our evaluation calls. The introduction of evaluation-related topics often took the form of requests for help in gathering data to identify potential program strategies rather than assistance in assessing program impact. Additionally, we had difficulty obtaining feedback from the CCIs about the evaluation tools and strategies that we had discussed in earlier meetings and in receiving requested information such as meeting minutes and phone logs that we had agreed to help organize and track. Eventually, the CCIs began to apologize for all of this, admitting that they were busy, that evaluation was not a priority, that their materials were not well written or organized, and that they were not able to recall what we had agreed to do.

Although considerable evaluation data was gathered for each project, we, as evaluators, ended up being more directive and acting more autonomously in this effort than had been originally envisioned. It became increasingly evident across the CCIs that there were differences of opinion regarding the design and operation of the projects, about the roles and authority of various project boards and other entities, and about the perceived function, importance, and value of the evaluation itself. Thus, the sense of partnership and joint decision-making that we had hoped to build was only minimally present throughout the project.

To probe this more fully, an informal survey was conducted of evaluation consumers. Although much has been written about the advantages and disadvantages of empowerment evaluation from the evaluator's perspective, little evidence has been gathered assessing how the approach is viewed through the eyes of evaluation consumers. Our questionnaire was 
distributed to key program coordinators and board members at each of the three CCI sites. The questionnaire addressed issues ranging from the CCIs' awareness of and interest in the provision of evaluation assistance, to their views on the potential roles and services of evaluators. This questionnaire was completed and returned by 19 of the CCI participants, or $63 \%$ of those surveyed.

The responses to the evaluation questionnaire mirrored the contradictions between the CCIs' verbal support of the evaluation approach and our subsequent difficulty with implementation of the approach. Of most interest was the striking lack of agreement among participants within and across the different projects about various aspects of the evaluation process. Nearly half of those participants surveyed, for instance, wanted us to act as external evaluators and to provide projects with a data-based "stamp of approval" that their projects were making a positive impact. Just over $25 \%$ wanted us to act more as evaluation consultants and to facilitate the program's own internal data collection efforts. This internal evaluation consultant role seemed more consistent with the empowerment approach we were envisioning. Interestingly, approximately $25 \%$ of the respondents did not identify the role they perceived or anticipated the evaluators would play. Further, although a need for evaluation assistance was generally acknowledged, a majority also felt that they were able to evaluate their projects independently, without external assistance. Thus, the way consumers viewed the very nature and purpose of the evaluation was remarkably variant across the different project participants.

Terms such as "process" and "outcome" evaluation were used by all participants. However, the way these terms were understood clearly differed among individuals. Participants often expressed an interest in process evaluation, but data perceived to satisfy this goal consisted primarily of descriptive information that simply documented program implementation. Participants also tended to endorse rather loose definitions of outcome achievement. For instance, overly subjective standards of proof were often utilized and programs were defined as successful or unsuccessful simply on the basis of the program director's "feeling."

Our examination of the stakeholders' perceptions and expectations provided us with some valuable insights into what we were experiencing and why. In addition, the information helped us define the kinds of questions it would have been helpful to stress in the early steps of the empowerment evaluation process.

\section{DISCUSSION}

Little is known about factors that may serve to make the empowerment evaluation approach more or less useful in different types of evaluation circumstances. Our experience provided us with some valuable lessons concerning some of these factors. Although many of these might also apply to other participatory evaluation approaches such as utilization-focused and stakeholder-based (see Patton, 1997a; Stake, 1980; Whyte, 1991), these lessons appear particularly relevant to the successful implementation of empowerment evaluation.

First, for the empowerment approach to be most effective, it may be critical to begin attempting to facilitate empowerment as early as possible in the evaluation process. In our particular case, the CCIs were not consistently involved in the initial request for or the selection of evaluators for their projects. Rather, the state had operated independently in securing contract services with us and then informed projects of our participation. Thus, our services were in some sense imposed on clients; this may have undermined the client's sense 
of control and "empowerment" in the earliest stage of the process. Furthermore, the contingencies for participation and the relevance of the resulting data may not have been made sufficiently clear to participants. For instance, the CCIs submitted grant evaluation updates independent of our involvement. The deadline for second-year grant applications also preceded the evaluation's completion deadline by four months. These actions may have suggested to the CCIs that continued funding was not completely dependent on clear empirical evidence of performance and effect. All of this may have lessened the CCIs' sense that evaluation was important or that they needed support in structuring an adequate evaluation plan.

Second, we may have failed to take into account how "stretched" many participants felt in the roles they were performing for the CCIs. In many cases, these roles were taken on in addition to other volunteer assignments and other more formal work commitments. Thus, the time and energy participants had to devote to evaluation activities were quite minimal.

Third, few structures were in place to support the long-term perspective required to set up methods to monitor results over time. For instance, participants perceived that significant changes in the availability of funding and prioritization of programs could occur with a shift in public interests or the election of new officials. Thus, many participants in the CCIs believed that program support might, at best, be temporary. In this environment, making the most of funding that exists today and doing what is perceived to be best for children and families in the short term often became the operative objectives and appeared to outweigh the commitment to long-range planning and thinking.

This difference in time perspectives also meant that there was some incongruence between the way we as evaluators and the CCI participants tended to define empowerment. We conceptualized it as an increase in participants' sense of control over and ownership of the evaluation process in the long term. Through this process, we hoped to build participants' capacity to sustain the evaluation efforts beyond the first year and, by doing so, to increase their ability to advocate effectively for their programs over time. CCI participants, on the other hand, appeared to define empowerment more in terms of the degree of enhancement they saw in their immediate short-term program advocacy functions, rather than as perceived increases in their capacity to build evaluation structures to meet long-term program accountability goals. In an analysis of the use of empowerment evaluation with different types of community grassroots organizations, Lackey, Moberg, and Balistrieri (1997) suggest that the goals of program advocacy and evaluation ownership may often collide. They found that the more evaluation participants were concerned with such issues as attracting funding and gaining recognition, the more they tended to want to turn the evaluation functions over to outside formal evaluators. In our case, there may have been similar tensions between the immediate need to promote program strengths and the ongoing, long-term need to gather data to fulfill program accountability requirements. This pressure appeared to be felt particularly by the state agencies. It was our perception that even though the state was promoting community-based decision-making, it seemed at times to feel pressured to justify its funding decisions.

Our experiences made us reconsider some of the basic premises underlying use of the empowerment approach in the context in which we were attempting to place it. In actual practice, what does it mean to accommodate to the needs and levels of expertise of one's clients? Whose standards of accountability should prevail in defining project outcomes, particularly if the clients' understanding and notions of what consists of valid measurable results are at variance with the standards endorsed by the evaluators? Can one have degrees 
of empowerment? For instance, we provided clients with a general evaluation framework and then asked them to tailor how they used this framework to best fit their own circumstances. Was there too much or too little structure provided to facilitate an effective use of the empowerment approach? How should the training agenda be controlled and what if the offered resources are not utilized? Finally, in what contexts and timeframes and with what types of supports will an empowerment evaluation approach tend to be more or less useful?

\section{RECOMMENDATIONS}

In this paper, we have focused not so much on whether the empowerment evaluation approach is "good" or "bad", but rather on its application in a specific comprehensive community context. Several points to consider emerged from this examination. Clearly, for an empowerment approach to be successful, stakeholders as well as evaluators have to be able to devote fairly significant amounts of time and energy to the evaluation process. Further, in addition to time, stakeholders must find it useful to commit to a level of responsibility for the evaluation effort that may be greater than that required in more traditional approaches. The scope and manner of this new mode of participation have not been made entirely clear in the existing literature.

These two points, time and level of responsibility, along with several other factors are best assessed and defined early in the empowerment evaluation process. Fetterman's first step, taking stock, appears to be the ideal opportunity to address a host of issues. It is imperative to begin by identifying all stakeholders in the particular project. Evaluators and evaluation consumers may need to think more broadly than they have in the past when identifying stakeholders. Obvious stakeholders include the program managers and funding agents. No less important are the program consumers, program staff, governing boards, affiliated state agency personnel, and technical assistance teams, and even the evaluators themselves.

In addition to identifying all stakeholders, it is important to assess the stakeholders' perceptions and understanding related to evaluation. It is essential to assess how the various stakeholders see themselves (and the evaluators) as participants in the evaluation. It may be particularly crucial to ask questions that address the roles and responsibilities of all participants. Among the issues to be discussed, agenda control and standard setting are critical. In terms of determining what outcomes should be investigated, Fetterman (1997) argues that defining meaningful outcomes should be a collaborative exercise. Greene (1997) and Scriven (1997) caution that all evaluation efforts involve some issues of power. Central among these are power issues: (1) whose questions are addressed in the evaluation, and (2) what criteria are used to make judgments about program success. Even if multiple stakeholders' views are included in setting evaluation objectives, it still may be difficult to represent all voices equally. In addition, there may be a dilemma regarding how to decide which stakeholders' views take precedence over others. One can also ask at what point the evaluator should impose professional standards of program evaluation (Guiding Principles for Evaluators, 1994) into the evaluation process.

Stock-taking must not only assess what types of outcomes stakeholders want from the evaluation, but also the stakeholders' working knowledge and understanding of evaluation processes and impact measurement. For those stakeholders with little evaluation knowledge, some sort of didactic pre-evaluation training could be helpful to offer before initiating this 
type of evaluation discussion. In addition, procedures should be established to guide the process of deciding how to proceed in the event of disagreements between the evaluators' and the stakeholders' perspectives concerning appropriate evaluation design and strategies.

Additionally, it is imperative to assess the external support structures and contingencies (or lack thereof) that support an empowerment evaluation approach. Support structures and contingencies that reinforce the importance of evaluation, the utilization of data to support program maintenance and continuation, and the use of evaluation frameworks to guide these data collection efforts in the long term appear to be essential to facilitating an empowerment evaluation approach. Not having these types of contingencies in place may lead some to consider evaluation to be a moot exercise. Thus, to be maximally effective, evaluation outcomes must be meaningfully linked to significant programmatic decisions, and the idea of "being empowered" must have real practical consequences.

As the evaluators facilitate the stock-taking process and introduce the empowerment evaluation approach, they may come to one of several conclusions. It may prove useful to view the implementation of an empowerment evaluation approach along a continuum. That is, some projects may be more amenable to such an approach than others. A given program may have sufficient resources (e.g., staff, financial), interest, motivation, and evaluation knowledge. Other programs may present barriers (insufficient staff resources, evaluation knowledge, etc.) that may preclude full implementation of an empowerment approach from the outset. Yet, these programs have sufficient interest in working with the evaluator toward an approach that becomes progressively more empowerment-oriented. And, still others may be a "good fit" for an empowerment approach in terms of knowledge and resources, but lack interest or motivation and therefore relegate evaluation to an external evaluator. Taking stock is critical to assessing the feasibility of utilizing an empowerment evaluation approach.

In sum, utilizing an effective empowerment evaluation model is a challenging task. Within the context of CCIs, participants with diverse skills and experiences find they have a common interest as stakeholders in a given project. The goal should be to attempt to utilize this diversity in a positive manner. As participant awareness and appreciation for such diversity increases, the diversity itself could serve as a springboard for discussion of different approaches to handling evaluation issues. In this way, better advantage might be taken of the varied backgrounds and perspectives of the evaluation consumers as well as the expertise of the evaluators. As Patton (1997a) suggests, the empowerment approach is a useful evaluation tool, but one that must be used thoughtfully and with great care if it is to be maximally effective.

\section{REFERENCES}

American Evaluation Association, Task Force on Guiding Principles for Evaluators. (1995). Guiding principles for evaluators. In W. R. Shadish, D. L. Newman, M. A. Scheirer, \& C. Wye (Eds.), Guiding principles for evaluators. New Directions for Program Evaluation, 66 (pp. 19-26). San Francisco: Jossey-Bass.

Ayers, T. D. (1987). Stakeholders as partners in evaluation: A stakeholder-collaborative approach. Evaluation and Program Planning, 10, 263-271.

Cousins, J. B., \& Earl, L. (1992). The case for participatory evaluation. Educational Evaluation and Practical Analysis, 14, 397-418.

Fetterman, D. M., Kaftarian, S., \& Wandersman, A. (Eds.). (1996). Empowerment evaluation: Knowledge and tools for self-assessment and accountability. Thousand Oaks, CA: Sage. 
Fetterman, D. M. (1996). Empowerment evaluation: An introduction to theory and practice. In D. M. Fetterman, S. Kaftarian, \& A. Wandersman (Eds.), Empowerment evaluation: Knowledge and tools for self-assessment and accountability. Thousand Oaks, CA: Sage.

Fetterman, D. M. (1997). Empowerment evaluation: A response to Patton and Scriven. Evaluation Practice, 18, 253-256.

Folkman, D. V., \& Rai, K. (1997). Reflections on facilitating a participatory community self-evaluation. Education and Program Planning, 20, 455-465.

Greene, J. C. (1997). Evaluation as advocacy. Evaluation Practice, 18, 25-35.

Kubisch, A. C. (1996-7). Comprehensive community initiatives: Lessons in neighborhood transformation. National Housing Institute [On-line]. Available: http://www.nhi.org/online/issues/85/ compcominit.html

Kubisch, A. C., Brown, P., Chaskin, R., Hirota, J., Joseph, M., Richman, H., \& Roberts, M. (1995). Voices from the field: Learning from comprehensive community initiatives. Paper presented at Roundtable on Comprehensive Community Initiatives for Children and Families of the Aspen Institute.

Lackey, D., Moberg, D. P., \& Balistrieri, M. (1997). By whose standards? Reflections on empowerment evaluation and grassroots groups. Evaluation Practice, 18, 137-146.

Murphy-Berman, V., Schnoes, C. J., \& Chambers, J. M. (in press). An evaluation model for assessing the effectiveness of comprehensive community initiatives: Three case studies in Nebraska. Evaluation and Program Planning.

Patton, M. Q. (1997a). Toward distinguishing empowerment evaluation and placing it in a larger context. Evaluation Practice, 18, 147-163.

Patton, M. Q. (1997b). Of vacuum cleaners and toolboxes: A response to Fetterman's response. Evaluation Practice, 18, 267-270.

Roberts, R. N., \& Wasik, G. H. (1996). Evaluating the 1992 and 1993 community integrated service systems projects. New Directions for Evaluation, 69, 35-49.

Ryan, K., Greene, J., Lincoln, Y., Mathison, S., \& Mertens, D. M. (1998). Advantages and challenges of using inclusive evaluation approaches in evaluation practice. American Journal of Evaluation, 19, 101-122.

Scriven, M. (1997). Empowerment evaluation examined. Evaluation Practice, 18, 165-175.

Sechrest, L. (1997). Review of empowerment evaluation: Knowledge and tools for self assessment and accountability. Environment and Behavior, 29, 422-426.

Stake, R. E. (1980). Program evaluation, particularly responsive evaluation. In W. B. Dockrell \& D. Hamilton (Eds.), Rethinking educational research. London: Hodeder \& Stoughton.

Whyte, W. F. (Ed.). (1991). Participatory action research. Newbury Park, CA: Sage. 